\title{
Secure GPRS Data Systems Based on 3D-Discrete Wavelet and Multiwavelet Transformations
}

\author{
Laith Ali Abdul-Rahaim \\ Electrical Engineering Department, Babylon University, Babil, Iraq
}

Email address:

drlaithanzy@yahoo.com

To cite this article:

Laith Ali Abdul-Rahaim. Secure GPRS Data Systems Based on 3D-Discrete Wavelet and Multiwavelet Transformations. American Journal of Embedded Systems and Applications. Vol. 3, No. 2, 2015, pp. 11-22. doi: 10.11648/j.ajesa.20150302.11

\begin{abstract}
This paper shows a new efficient data delivery method based on three dimensional discrete Multiwavelet transformations Critically Sampled (3D-DMWTCS). This method was been done by get the data from any data source then catted the stream data to a packets of A certain length then each of these packet will convert to a square matrix, and then collected these square matrices in three dimensional matrix form. The 3D - discrete Multiwavelet transforms Critically Sampled (3D-DMWTCS) will be applied to each three dimensional matrix form and the output of this procedure will be also in three dimensional matrix forms and contain 3D-DMWTCS coefficient as real numbers. After this stage, the 3D matrix will be converting to square matrix and then to serial data stream of samples, then will be sending through USB port to SIM908 GSM GPRS module that must secure a connection between two or more communication points. As well as this encryption method can be done using three dimensional discrete wavelet transformations (3D-DWT) and (2D-DMWTCS) and (2D-DWT). The purposed of these methods is to maintain the confidentiality of the information sent, whether it is written texts, or data, or talk speech, and it is done with a high data rate and excellent coding. It is impossible or difficult to decrypt these methods without knowing how these encryption systems are build. The decryption is done by extracting the encrypted data from the Multiwavelet decomposition vector using the algorithm of inverse of 3D-Discrete Multiwavelet Transformation. The encrypted messages that send to other points consists 3D-DMWTCS decomposition vector. The encryption key that used in this method is 3D-DMWTCS method so the decryption key will be 3D-IDMWTCS. Due to the GSM GPRS mobile communications systems mostly use OFDM technology, therefore these technology has been used in the designed of wireless communication transceivers system using MATLAB2014a to simulate GSM GPRS under multichannel types and run the simulations to determine the specifications of these cryptographic systems under various conditions. The simulation results proved that these methods are retrieving the security and improve the performance of the communications system.
\end{abstract}

Keywords: 3D-DMWTCS, 3D-DWT Cryptography, GPRS, OFDM, SIM908, UDP, TCP

\section{Introduction}

The implementation of voice, SMS and MMS services into wireless communication system. The main aim of this work focus on using mobile devices, for example tablets and smart phones, to access to multimedia video files and call control session. In an internet-to-mobile data transmitting scenario, the protocol of services employs user datagram protocol (UDP) as their transport protocol [1]. If UDP Compared with transmission control protocol (TCP) [2], the UDP does not fructify retransmission delay that makes TCP protocol captivating to delay responsive applications. All packets of UDP contain a header and payload. UDP also have a cyclic redundancy check (CRC) to catch the integrity of packets; therefore, it can detect any error in the packet header or payload. If detected an error, the packet is discarded and declared the error. UDP packet transmission in internet is best efforts by network congestion case yields packet loss. At the receiving side with certain data rate (DR) packets are either exact detect or lost.

The physical layer (PHY) and medium access control (MAC), and the current channel status, are usually small compared to the packet length. In the applications of wireless systems like third-generation $(3 \mathrm{G})[3,4]$, which needed low and medium DR, each frame in physical layer corresponds to a transmission unit [5]. To associate high DR services, MAC protocol specifies that radio link protocol (RLP) can partition each physical layer frame into smaller logical frames named logical transmission units (LTUs), each associated with a 16 
bits of CRC [6]. The LTU size may be change between [300 $600]$ bits. After stage of channel decoding in the receiver side, the residue error will be detected by CRC. This information of frame error is will be available at the RLP layer. Also one can combine the link adaptation with FEC coding at the packet level to get maximum flexibility. But, this way culminate from signaling significant level, delay and complexity [7]. When TCP is used, packet loss will be recovered by using congestion control. The TCP/RLP has been shown in Details in $[8,9]$. User datagram protocol (UDP) is part of a miniature TCP/IP suite. The UDP provides any applications full transport layer services. Fig.1 (a) shows UDP in the TCP/IP suite model and it is clear that is located in the transport layer. UDP can be establishing a link or a connection between any two processes at begins and the ends of any data transmission [10]. The transmission of data will done with minimal overhead, without using acknowledgment or flow control of received data. The minimal error control was done by neglect (discard) the received packets which contain error in some bits using checksum test.Fig. 2 shows the comparison between TCP and UDP addressing process which defines each domain of its protocol $[11,12]$.
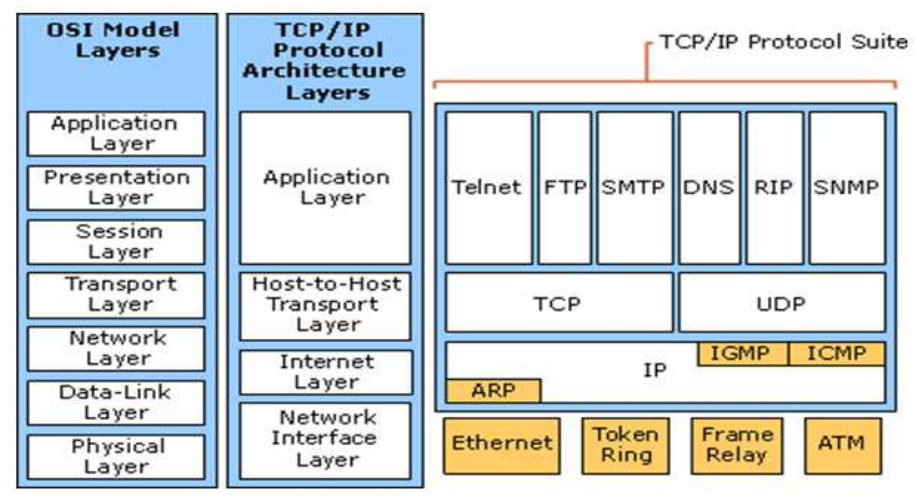

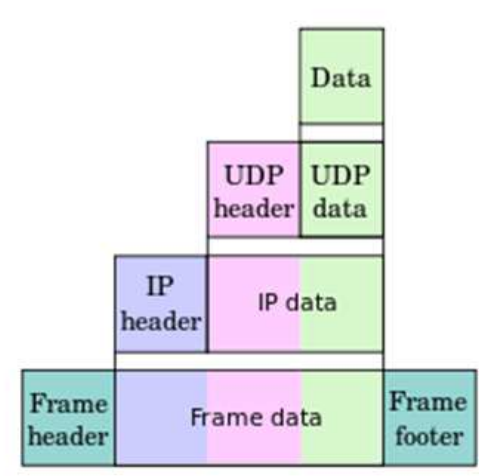

Application

Transport

Internet

Link

Figure. 1. TCP/IP suite model show UDP and TCP/IP.

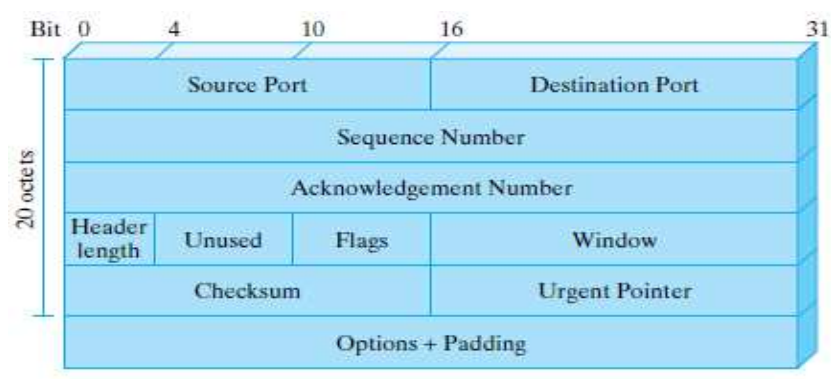

(a) TCP Header

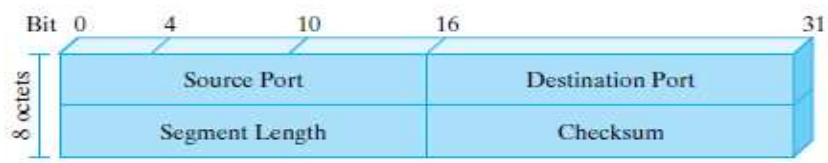

(b) UDP Header

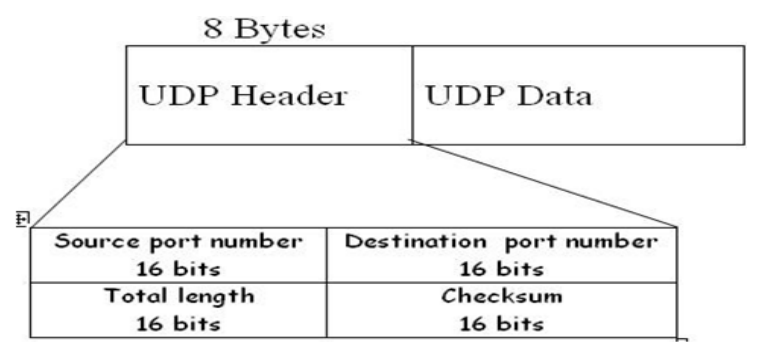

(c)

Figure. 2. a) TCP Header, b) UDP Header, C) User datagram format added to the UDP datagram.

\section{The Proposed System Theories}

To establish wireless communication between any two points will be the use of sim908 based on ARM926EJ_S core. SIM908 work at $156 \mathrm{MHz}$, and has redundant MIPS to run programs other than telecommunication protocols [1]. So SIM908 can be integrated into a wide range of applications. Embedded AT is for fully utilizing SIM908 resources, providing interfaces to move some external MCU functions into itself, so as to save customer's cost. SIM908 module is perfect Quad-Band GSM/GPRS modules that integrate GPS technology for satellite navigation $[13,14,15,16]$. The built-in design that incorporated GPRS and GPS in a SMT package will clearly save both time and costs for users to promote GPS enabled applications. The standard industry interface and GPS function let variable dominions to be tracked easily at any place and time if the signal covering the area. The AT commands used to make a connection between sim908 GSM modules are summarized in table 1 . The procedure to establish a connection between two or more points using TCP or UDP protocol with sim908 GSM modules will be according to the following steps are summarized in Fig.(3), Fig.(4) and Fig (5).

Table 1. AT commands used with sim908 to make connections.

\begin{tabular}{|c|c|c|}
\hline AT command & Response & Description \\
\hline $\mathrm{AT}+\mathrm{CIPMUX}=$ & OK & $\begin{array}{l}\text { Selects single connection }(0) \text { or } \\
\text { multiple connection }(1)\end{array}$ \\
\hline $\mathrm{AT}+\mathrm{CSTT}="$ myAPN" & OK & Sets APN \\
\hline $\mathrm{AT}+\mathrm{CIICR}$ & & Brings up wireless connection \\
\hline $\mathrm{AT}+\mathrm{CIFSR}$ & & Get local IP address \\
\hline AT+CIPSTART & & $\begin{array}{l}\text { Establishes a connection with a } \\
\text { server. }\end{array}$ \\
\hline $\mathrm{AT}+\mathrm{CIPSEND}$ & & $\begin{array}{l}\text { Sends data when the connection is } \\
\text { established. }\end{array}$ \\
\hline $\mathrm{AT}+\mathrm{CIPCLOSE}$ & & Closes the connection \\
\hline
\end{tabular}



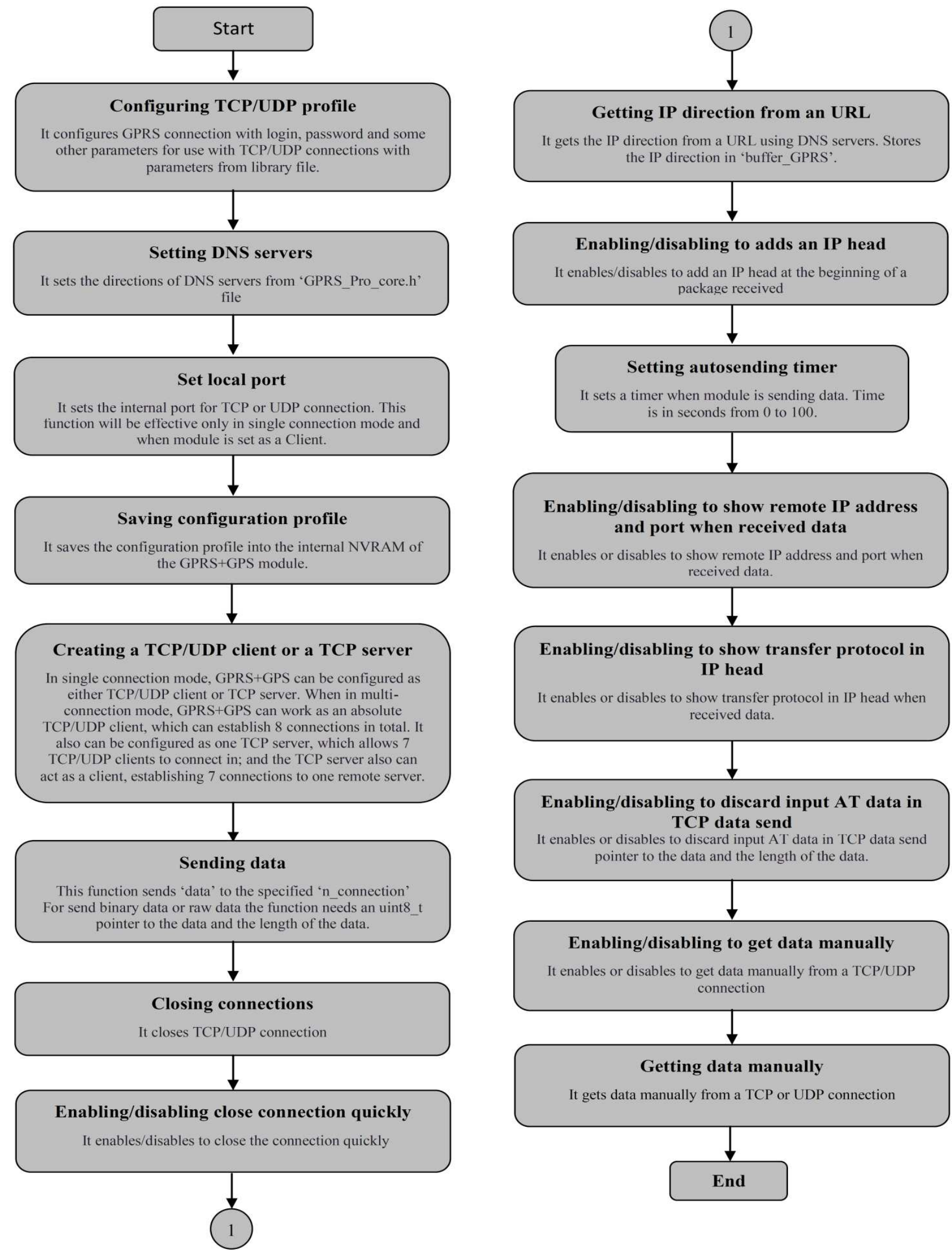

Figure. 3. Flow chart of the Procedure to establish connection between sim 908 modules using TCP or UDP. 


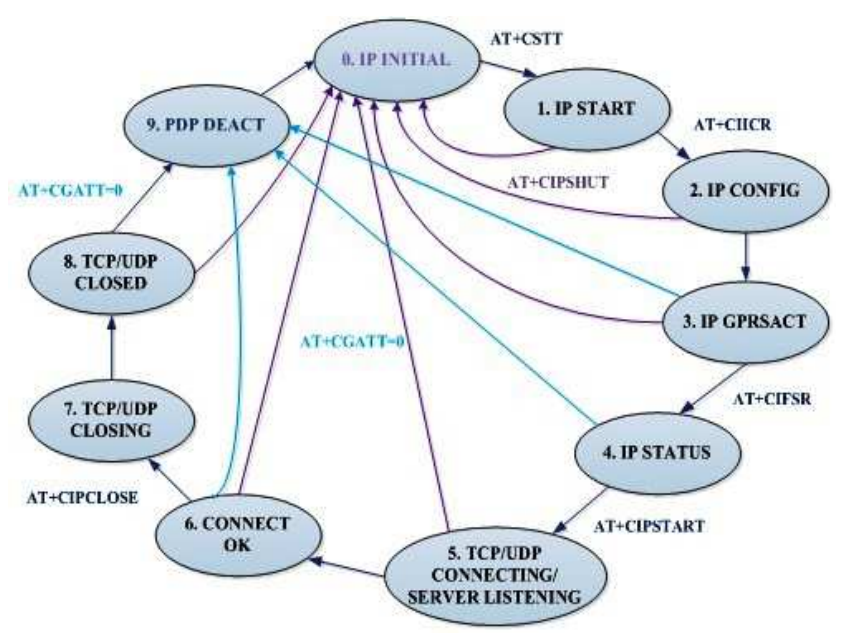

Figure. 4. The states diagram for single connection for sim 908.

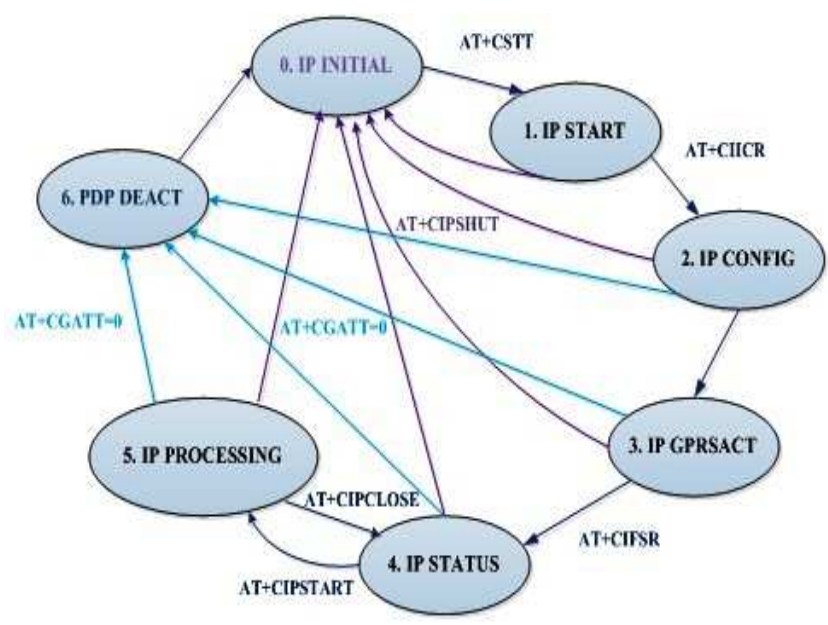

Figure. 5. The states diagram for multi connection of sim 908 .

\subsection{Computation Method of 3D-DWT and 3D-DMWTCS}

Discrete Wavelet and Multiwavelet transform given a good indication in applications of signal processing. Recent work on Multiwavelet have been studies the basic theory, methods of constructing new multifilters and the denoising and compression applications in of video and image [17, 18, 19, 22].The computation methods of 1,2 and 3D of DWT are shown in $[17,20,22,23,24,25]$ also 1 and 2D of DMWTCS are shown[18,24,25] and 3D-DMWTCS shown below.

\subsubsection{A Proposed Computation Method of 3D-DMWTCS}

The algorithms for computing three dimensional discrete Multiwavelet transform Critically Sampled (3D-DMWTCS) have been described in this section in a simple and easy way to verify procedure using matrix multiplication and addition. To compute 3D-DMWTCS one must know how compute 1, 2Ddiscrete Multiwavelet transforms Critically Sampled and its inverse 1,2D-IDMWTCS. In 3D- discrete Multiwavelet transformation Critically Sampled algorithm is defined in 3D, so the transformation procedure will done successively in $\mathrm{x}$-, $\mathrm{y}$ - and z-directions.
For a 2D-DMWTCS, the procedure was applied to each vector in $\mathrm{x}$-direction first, and then to each vector in y-direction. Similarly, in 3D- discrete Multiwavelet transformation Critically Sampled the procedure is defined in $3 \mathrm{D}$ and the transformation algorithm is applied successively in $\mathrm{x}-$, $\mathrm{y}$ - and $\mathrm{z}$-direction.

Let's take a general 3D signal, for example any NxNxM matrix. The computation 3D-DMWTCS need the following procedure:

1. construct $3 \mathrm{D}$-matrix A to represent the $3 \mathrm{D}$ input signal,

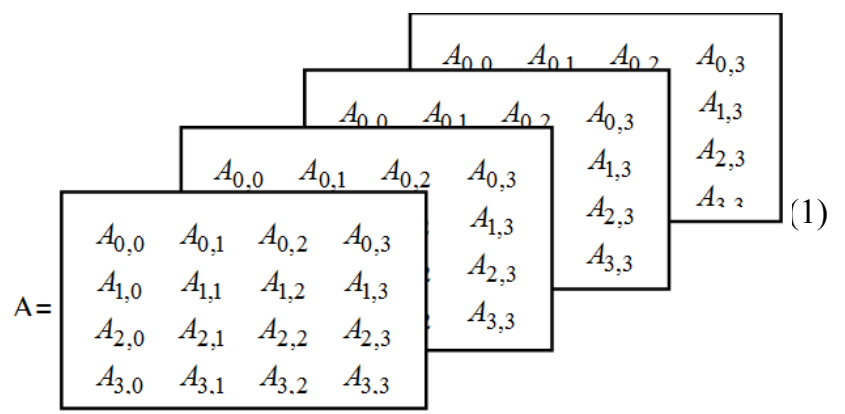

2. Using 2D DMWTCS algorithm to each $\mathrm{NxN}$ input matrix, which result in a $\mathrm{Y}$ matrix $(\mathrm{NxNxM})$.

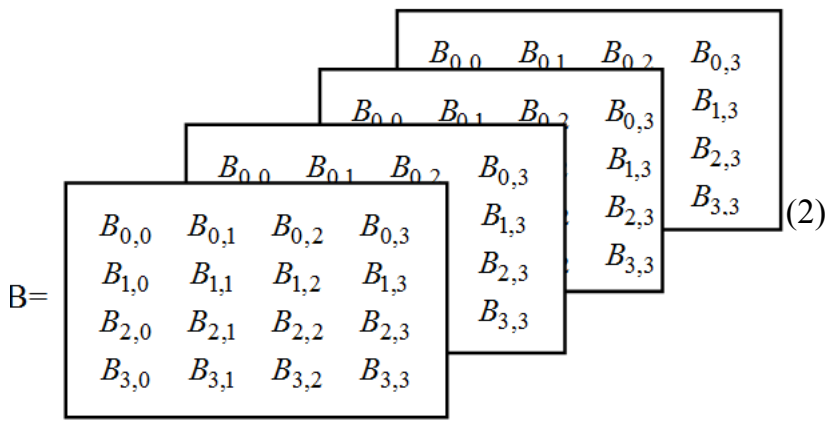

3. Using 1D-DMWTCS algorithm shown in appendix (A) to compute 1D-DMWTCS to each element of $\mathrm{NxN}$ matrix in all $\mathrm{M}$ matrices in $\mathrm{z}$-direction, which can summarized as follows:

a. For each i,j element in the $1^{\text {st }}$ matrix construct a vector of $\mathrm{Mx} 1$ for each element in $\mathrm{z}$-direction output matrices from 2D-DMWTCS in step 2, this operation is done as below:

$V(i, j)=\left[\begin{array}{llll}B_{i, j, 0} & B_{i, j, 1} & B_{i, j, 2} & B_{i, j, 3}\end{array}\right]_{1 \times M}^{T}$

Where $i, j=0,1,2, \cdots, N$

b. Applying 1D-DMWTCS algorithm to each the construct vector $V(i, j)$.

4. Repeat step 3 for all construct vector $\mathrm{i}, \mathrm{j}$.

5. Finally, aNxNxM of 3D-DMWTCS matrix results from the NxNxM original matrix using 3D-DMWTCS.

\subsubsection{Computation Algorithm of 3D-IDMWTCS}

The original 3D signal can be reconstruct from the 
transformed 3D-discrete Multiwavelet transformed Critically Sampled signal, by applying the inverse discrete 3D- discrete Multiwavelet transform (3D-IDMWTCS) algorithm. The Computation of 3D-IDMWTCS for 3D Signal can be summarized in the following procedure

1. Let $\mathrm{X}$ be the $\mathrm{N} \times \mathrm{N} \times \mathrm{M}$ the 3D-Discrete Multiwavelet transformed using critically sampled scheme matrix

2. Built the reconstruction transformation matrix $\mathrm{M} \times \mathrm{M}$.

3. Apply 1D- IDMWTCS algorithm shown in [10] to each construct a vector of $\mathrm{M} \times 1$ of $\mathrm{N} \times \mathrm{N}$ in the $1^{\text {st }}$ matrix all $\mathrm{M}$ matrices in $\mathrm{z}$-direction.

4. Repeat step 3 for all construct vector $\mathrm{i}, \mathrm{j}$

5. Built reconstruction transformation matrix $\mathrm{N} \times \mathrm{N}$.

6. Apply 2D- IDMWTCS algorithm to each $\mathrm{N} \times \mathrm{N}$ matrix result from step 3 in the $\mathrm{z}$-direction.

7. Finally, a $N \times N \times M$ matrix results from the transform matrix $\mathrm{N} \times \mathrm{N} \times \mathrm{M}$ to represent original matrix using 3D-IDMWTCS algorithm.

\section{Proposed Secure Wireless Transceivers}

The proposed secure wireless transceivers using TCP or UDP protocol is shown as in Figure (6). Each transceiver need to laptop and sim908 GSM module. The Matlab 2014a are used to build a Simulink function to represent the proposed secure function based on 3D-DWT or 3D-DMWTCS functions and there inverse. These functions build as Matlab function using M file and these function are call by the Simulink's (S-function) as shown in Fig (7). These functions will compute 3D-DWT or 3D-DMWTCS coefficients and then send to anther function that does OFDM modulation after convert the $3 \mathrm{D}$ to $2 \mathrm{D}$ matrices, and then these 2D matrices to vectors of $1 \mathrm{D}$ then configures these vectors to packets that will be feed to OFDM function. The output of OFDM packet convert to serial and feed to the USB port using UDP or TCP protocol to sim908 and through $\operatorname{sim} 908$ to anther sim 908 on the other side that will reflect the process that took place in the transmitter.

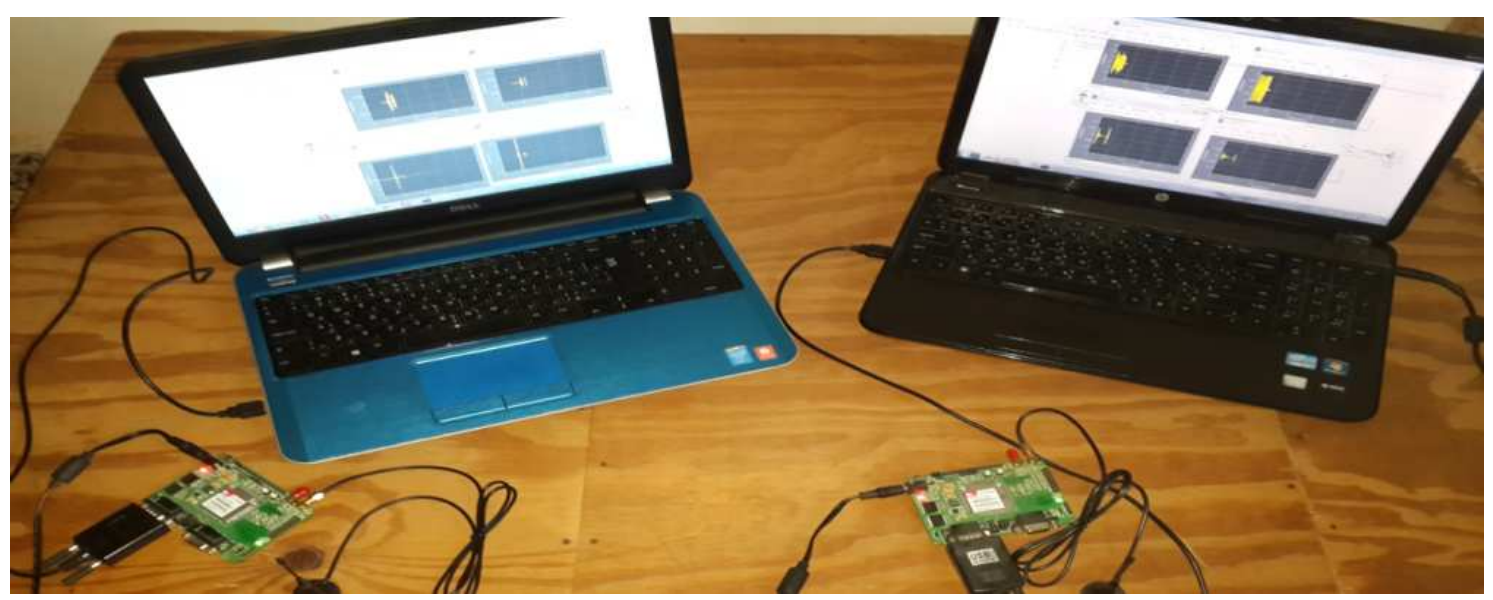

Figure. 6. The real picture of proposed transceivers system based on sim 908 .

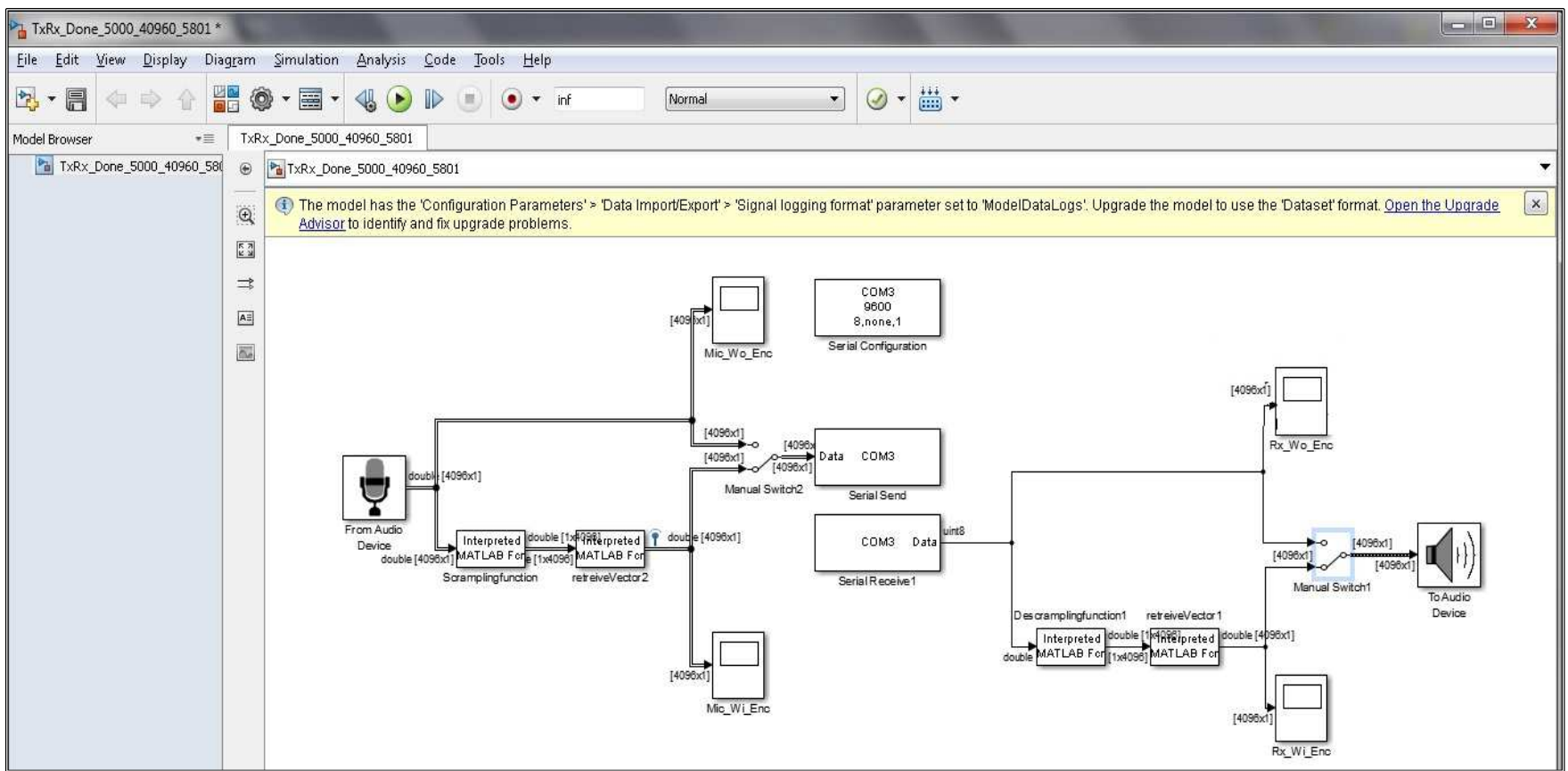

Figure. 7. The proposed transceivers system design based on Simulink. 
At the receiving end, all the steps of proposed algorithm applied but in reversely order to retrieve the original data. However frames are divided into 4096 samples to offer 16 ! × 16 ! Possible probability permutation for each 2D-Matrices. This changeability of the Encrypted signal is increased to be $(16$ ! $\times 16$ ! $\times 16$ !) when interring the total frame permutation (the third dimension permutation) is also performed. Not all permutations give good security quality so the effective permutation used [6]. Fortunately, results offer high security level to the system. The block diagrams of the proposed Encryption and decryption based on this new scheme are shown in Fig.(8).

Although using the subject scheme, in order to have insight of time and frequency domain analyses, the recorded wave files of the various speech segments are implemented and evaluated using Simulink MATLAB ${ }^{\circledR}(\mathrm{R} 2014 \mathrm{a})$. The used of MATLAB ${ }^{\circledR}(\mathrm{R} 2014 \mathrm{a})$ approach for software simulation offers many important advantages in the described applications. Thus, modeling and simulation flexibility is greatly enhanced.

To reinforce the quality of the obtained results, the experiments are conducted not only in English but in Arabic languages as well.

Pauses between talk burst cannot be sensed and there is no residual intelligibility.

In term of security system, the concept of the residual of intelligibility while the quality of the recovered data are subjective quantity, thus the scramble and descramble process techniques are estimated on the results of expert listeners during the test $[22,23]$.

Table 2. Purposed System Specifications.

\begin{tabular}{ll}
\hline Parameter & Range \\
\hline Input speech & $300 \mathrm{~Hz}$ to $3400 \mathrm{~Hz}$ \\
Sampling frequency & $44100 \mathrm{~Hz}$ \\
Type of transformation & 3D-Discrete Wavelet Transform \\
Frame length & 3D-Discrete Multiwavelet Transform \\
Frame duration & 4096 sample \\
Total Changeable coefficients & $92.879 \mathrm{~m} \mathrm{sec}$ \\
\hline
\end{tabular}

The tests were made tighter by adopting the following steps:

1. Separating digits that are pronounced via male and female as well.

2. Examinations are applied for digits also sentences are included too.

3. Those segments also are proved by male and female too.

4. However, to have more practical results, exchanged segments are carried out via two languages for male and female individually. The results are calculated in terms of correctly identified words $\mathrm{I}_{\mathrm{Q}}$, which is equal to $\mathrm{Q}$ :

$$
\mathrm{I}_{\mathrm{Q}}=\frac{\mathrm{E}}{\mathrm{T}} \times 100 \%
$$

Where $\mathrm{E}$ is the difference between the right words, and the wrong words and $\mathrm{T}$ is total words $[10,15]$.

The transmitter sends data in plaintext or clear text; the process of disguising so that the information is hidden is called encryption. The encrypted data is referred to as cipher text. When receiver performs the reversal of the encryption processes, this is called decryption. The terms "encipher" and "decipher" are synonymously used for "encrypt" and "decrypt" $[5,6]$. The security of proposed algorithm is based on how the way of the algorithm works as secret key, and named as restricted algorithm. Restricted algorithms have historical interest system.
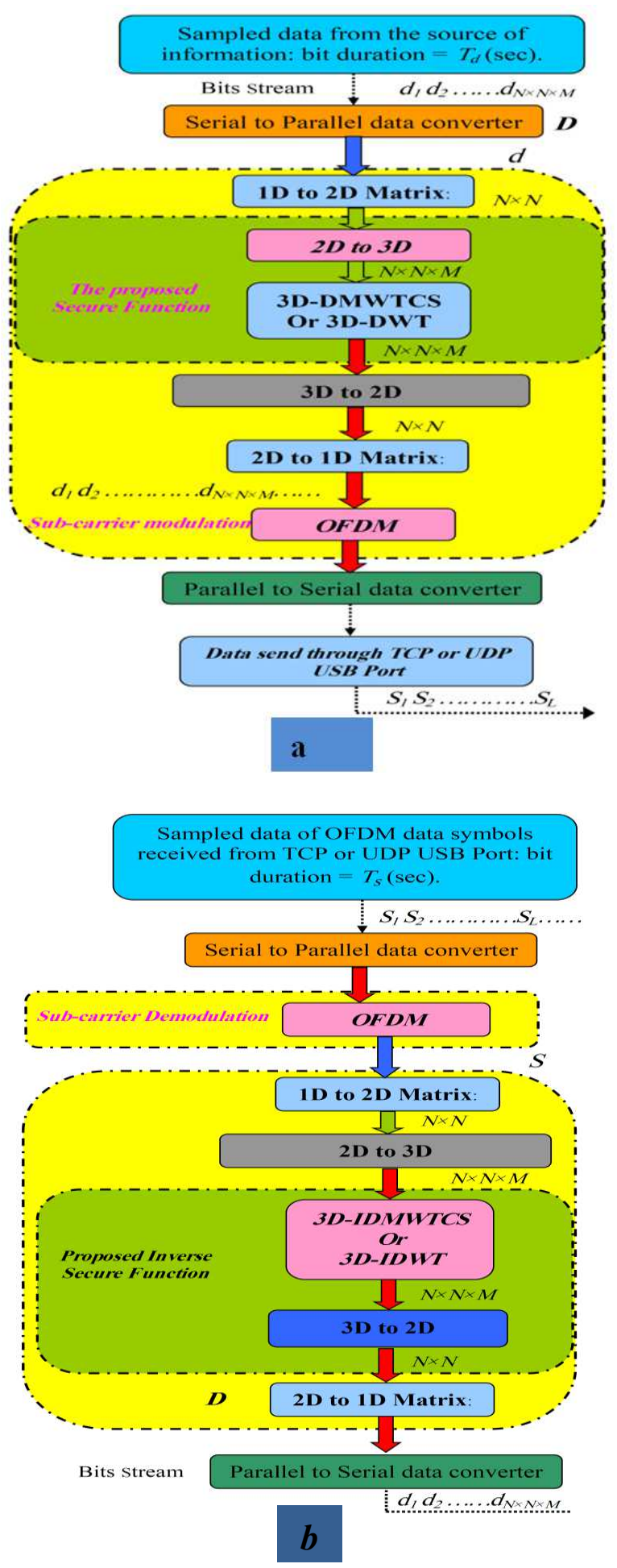

Fig. 8. Encryption algorithm at : a)Transmitter, b) Receiver. 


\section{Real Time Results of the Proposed System}

The below graphs (Fig. 9 to Fig. 16) shows the results of real time results based on GSM network and sim908 using time and spectrum expressions of Simulink. And GUI of SMS from sim908 to anther sim908 with Simulink over GSM network with and without using 3D-DMWTCS proposed encryption method in receiver side.

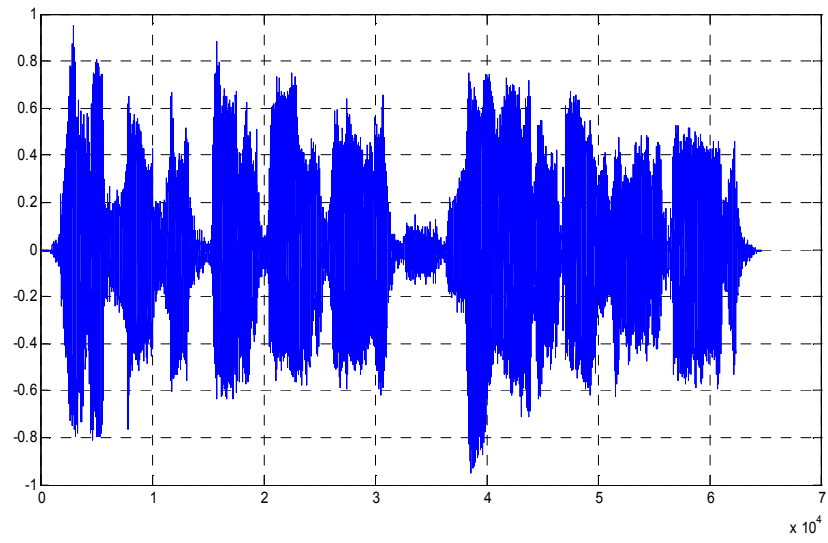

Figure 9. Transmitted speech over GSM network without encryption.

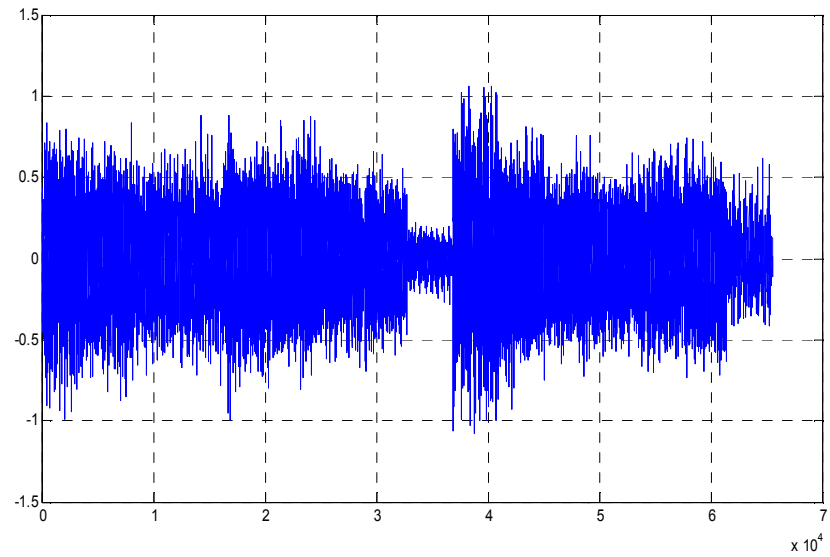

Figure 10. Transmitted data over GSM network with encryption.

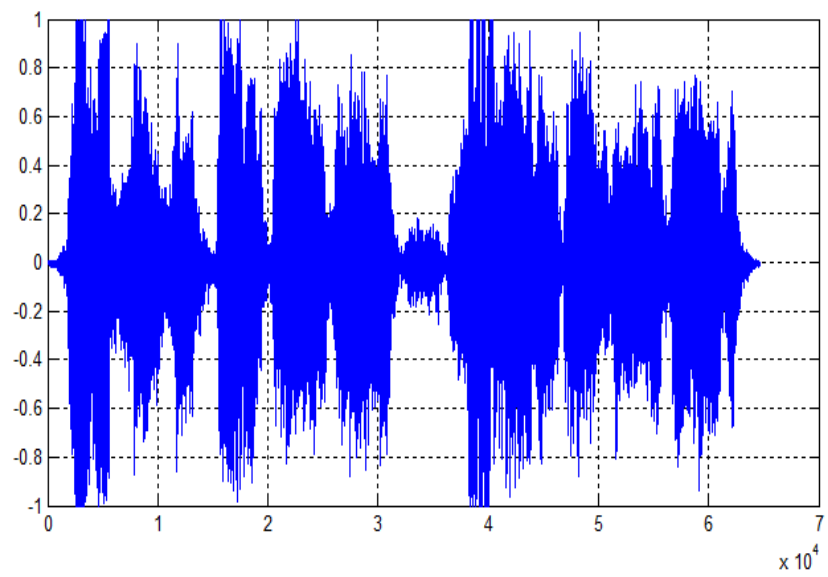

Figure 11. Received data over GSM network without decryption.

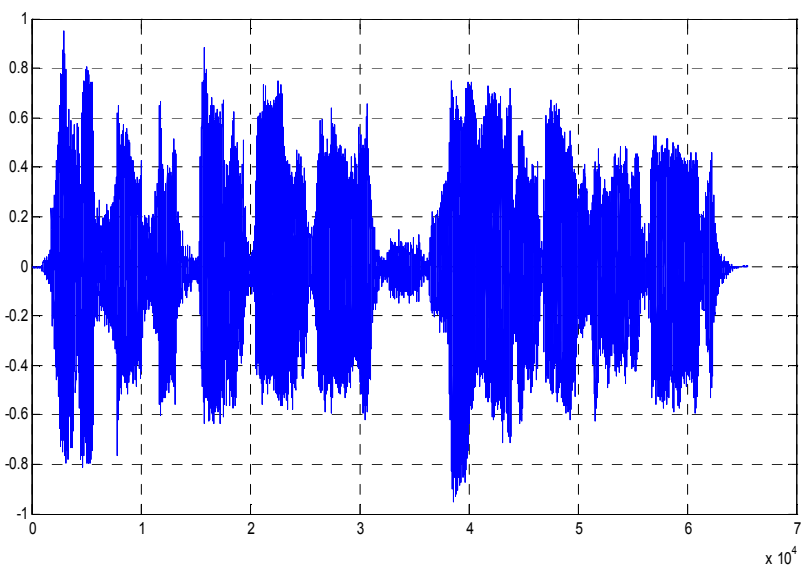

Figure 12. Received data over Ad Hoc network with decryption.

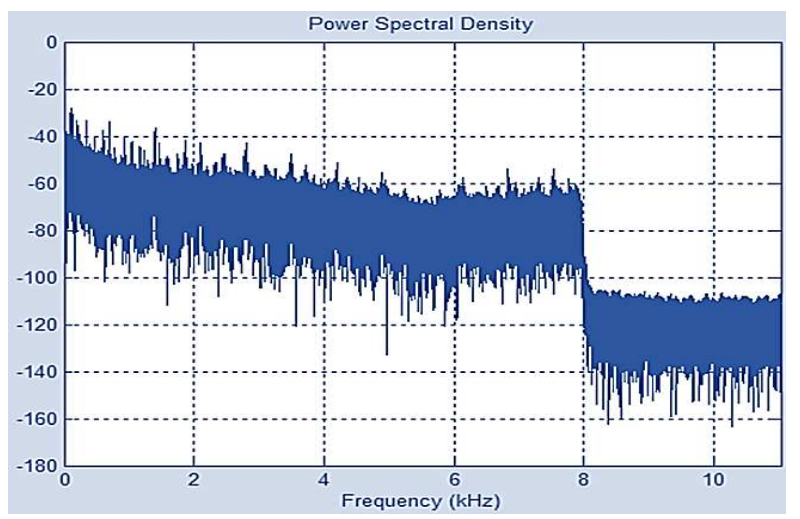

Figure 13. Power Spectral Density of Audio wave.

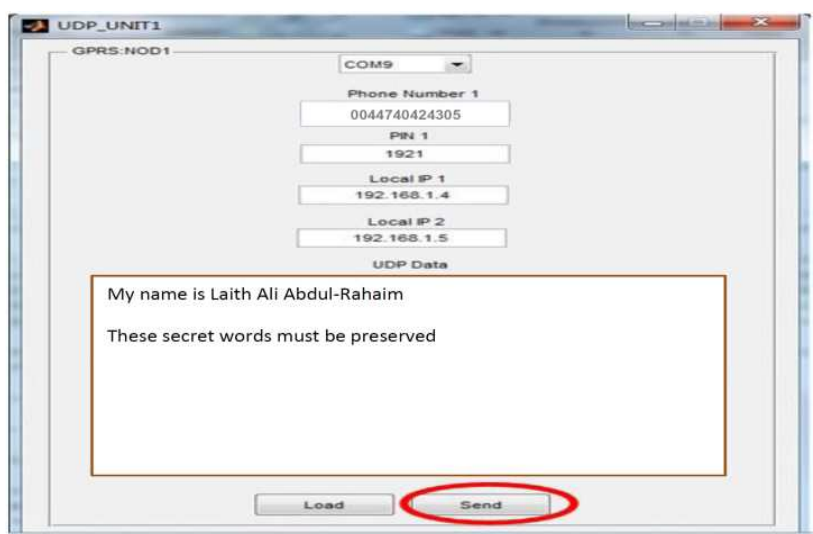

Figure 14. Transmitted SMS over GSM network with encryption.

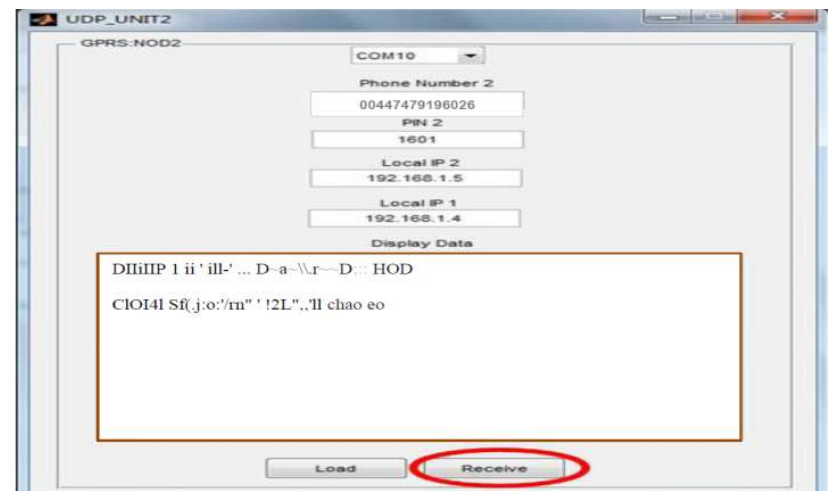

Figure 15. Received SMS over GSM network without decryption. 


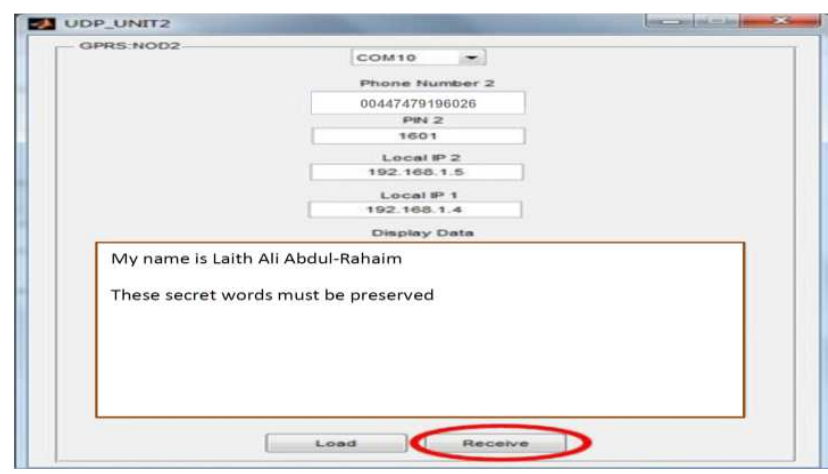

Figure 16. Received SMS over GSM network with decryption.

\section{Performance Enhancement in Simulation Results}

In this section, the contents show the simulation results of OFDM with proposed encryption based 3D-DMWTCS and

\section{D-DWT method.}

However, for time-domain it is clearly represented as discrete-time signals. In frequency domain the division of energy is not as original as before the encryption process application. The spectrum is reversed altogether which inverts the distribution of energy level with respect to function of frequency. However, the data is mixed in frequency domain which is similar to convolution in time domain. While transmitted signal is represented in time-domain this leads that any unauthorized access that tries to de-ciphering the data without knowledge about the used scheme, would have to convolve in time-domain which, without doubt would be time consuming process based real-time systems. Furthermore, non- knowledge the permutation order of the system that's why he would have to apply on each frame could be recognized to take infinite time [20,21] as shown in fig.(17).

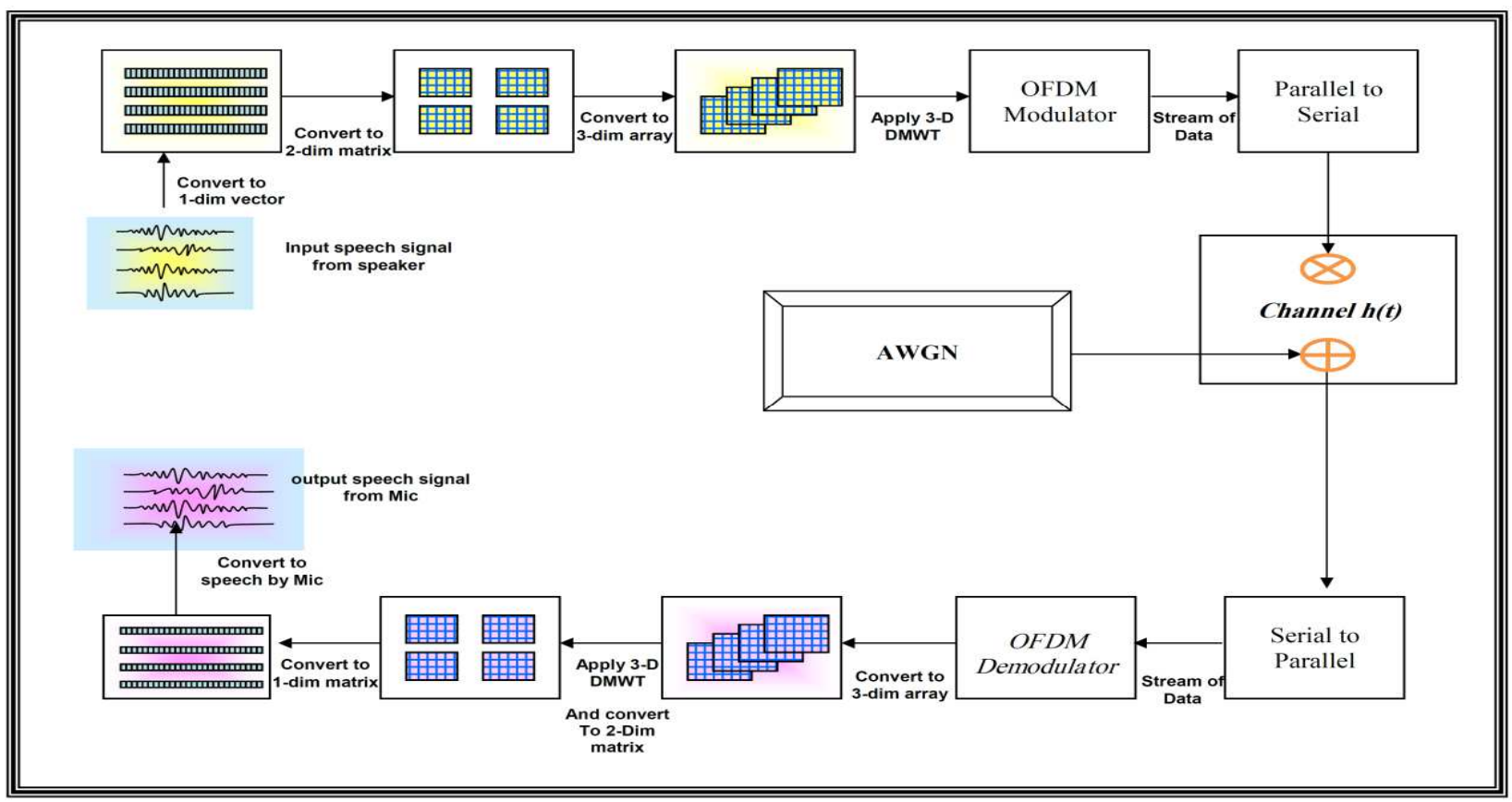

Figure 17. Block diagram of real time encryption system.

These parameters are shown in table (2)

Table 3. Simulation Parameters.

\begin{tabular}{ll}
\hline Bandwidth & $\mathbf{2 5 0 k H z}$ \\
\hline & AWGN \\
Channel model & Flat fading+AWGN \\
& Frequency selective fading+AWGN \\
Delay spreading $\left(\mathrm{T}_{\mathrm{d}}\right)$ & $0.1 \mu \mathrm{sec}$ \\
FFT Points & 64 \\
Symbol Number & 26 \\
\hline
\end{tabular}

After an extensive tests the results showed over a long period to create those tests clear for listeners, the speech files of that contain wave signals are played and listened by listeners. By following mechanism steps, thirty listeners who are all listened to 50 encrypted wave segments. Segment consists of the digits 0 to 9 is spoken in cluster of four digits. Additional, tests are not restricted to spoken digits only but also to sentence segments. So as to make test stringent and result has oriented feature, the test was hard and consumes time, and the tests are implemented in English as well in Arabic language. However, duplication of spoken digits of the same position is avoided. The tests were inflexible by using:

i. Separating the digits that were spoken by male and female as well.

ii. Test is done for not limited to digits only but also for sentences. The test segments also recorded.

iii. The recorded segments are tested via two languages by male and female.

The recorded data file, contains spoken digits "Zero, One, 
Two" vocal by a male is showed in. Figs. (18) - (20) which represent time-domain representation of original ciphered data and deciphered files respectively. On the rest, Figs (21) - (23) reveal distribution of power as a function of frequency of original encrypted and retrieved speech, respectively.

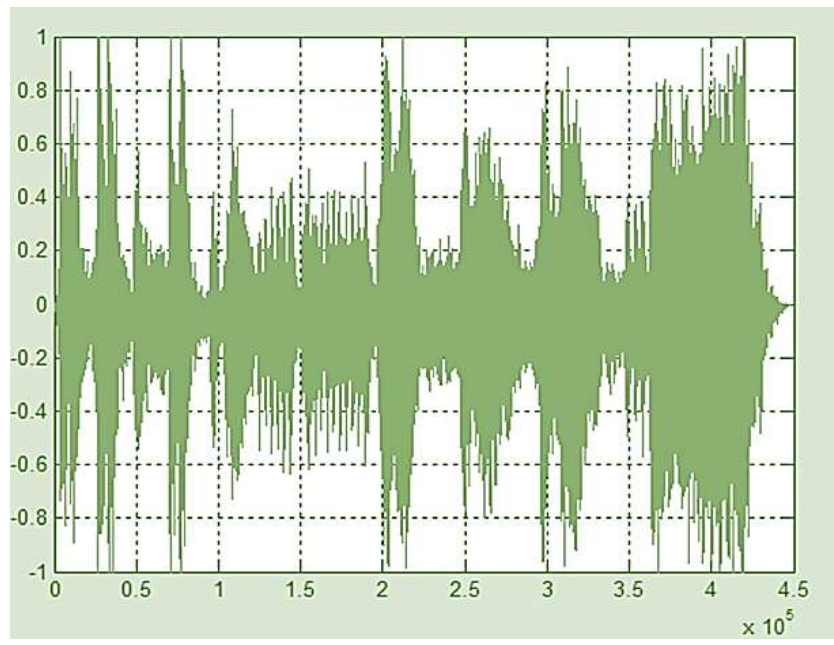

Figure 18. The original Audio File in time Domain.

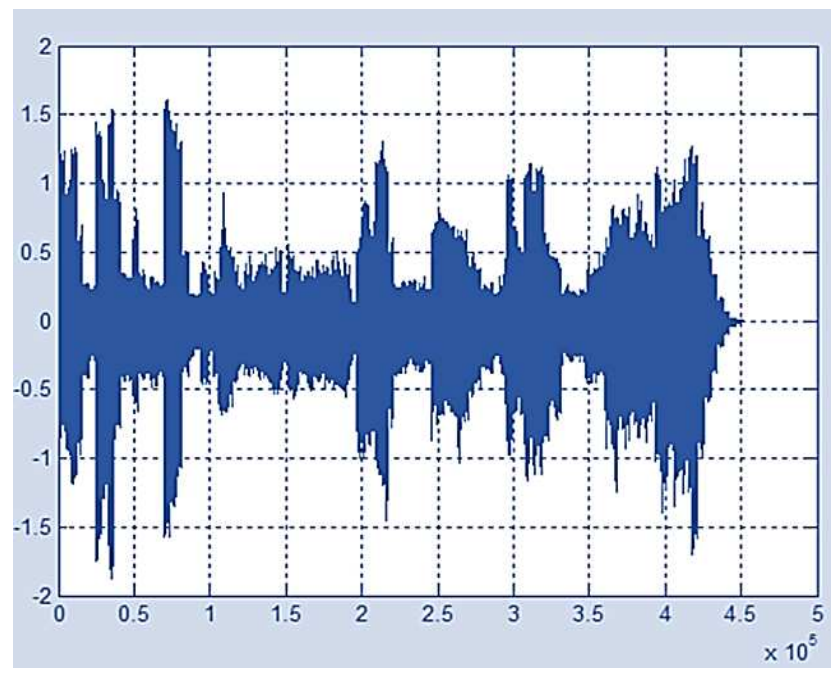

Figure 19. The 3D-DMWTCS encrypted Time Domain of Original Audio File.

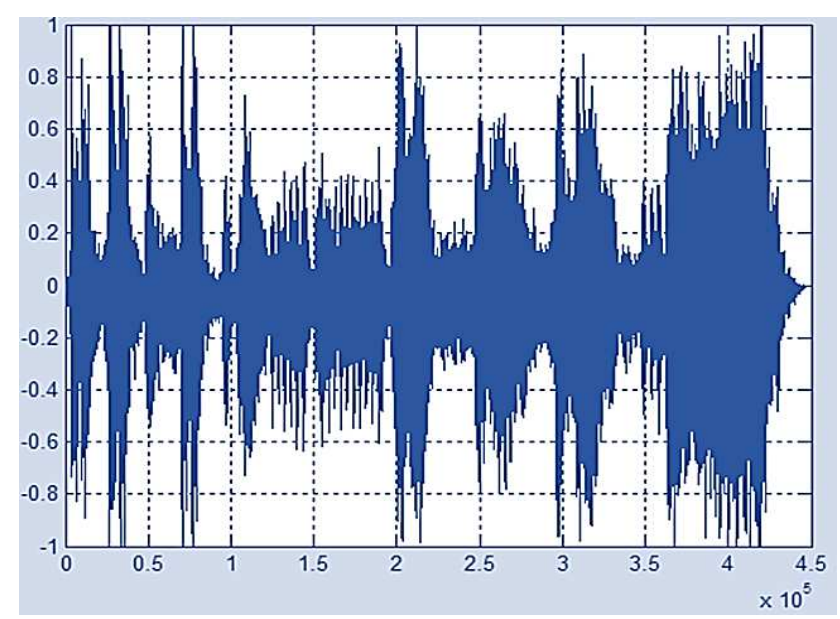

Figure 20. The 3D-DMWTCS decrypted Time Domain of Recovered Audio wave.

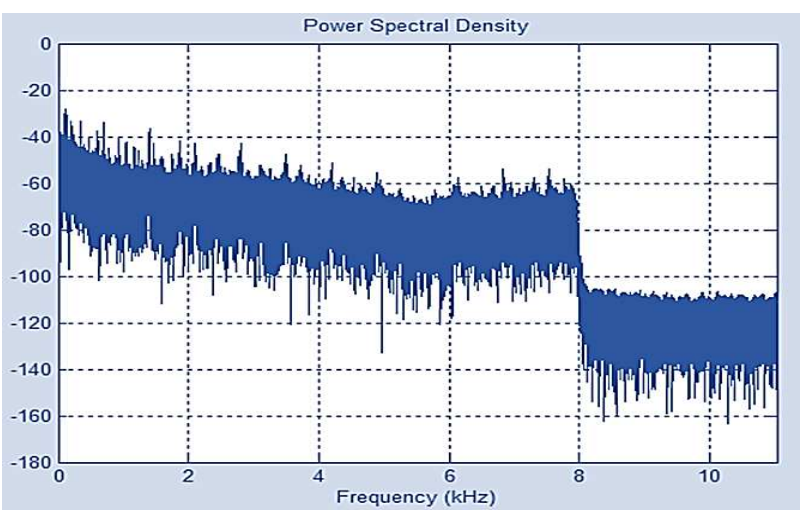

Figure 21. Original Power Spectral Density of Audio wave.

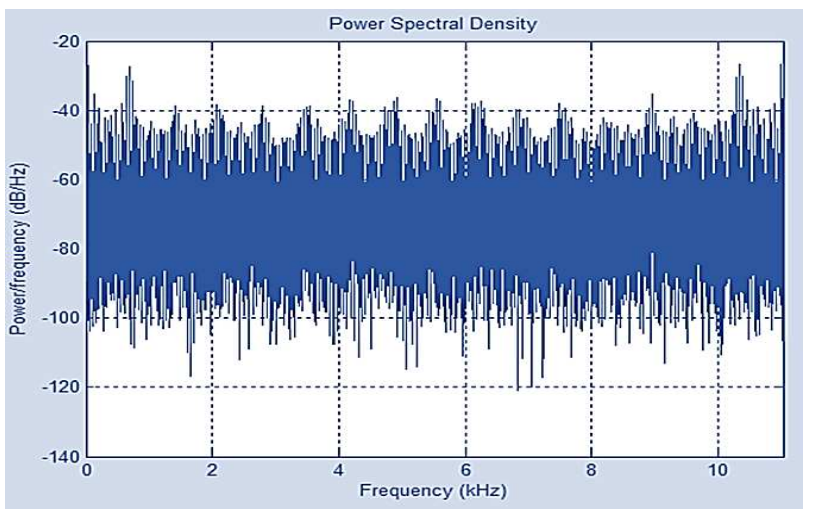

Figure 22. 3D-DMWTCS Encrypted Power Spectral Density of Audio wave.

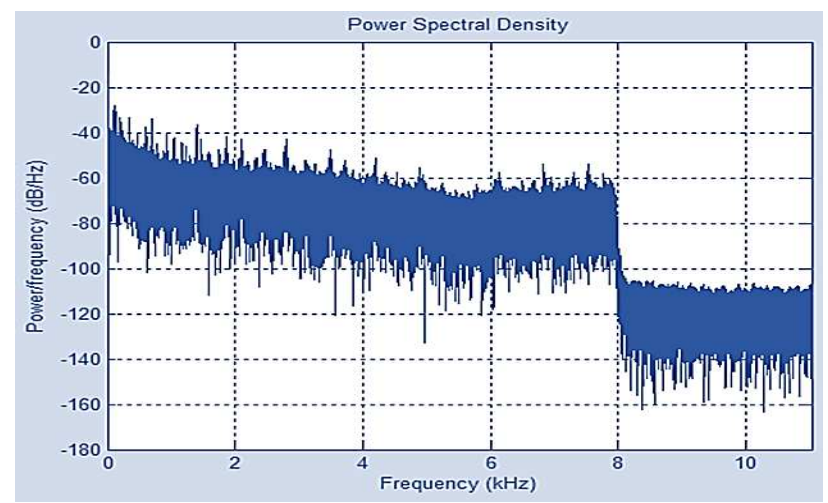

Figure 23. Recovered of 3D-DMWTCS Encrypted Power Spectral Density of Audio wave.

\subsection{The Encryption-OFDM in AWGN Channel}

The MATLAB V8.1 is used to simulate the Encryption OFDM transceiver proposed system as shown in Fig.(17). Most MATLAB functions are written to simulate the encryption system as shown in Fig.(17). The functions include frame resizing, Encryption-description, the using of pilot carriers, etc. the output of the simulated proposed system is estimated and represented in Fig. (24), and gives the BER performance of the Encryption-OFDM using discrete wavelet and Multiwavelet transformation in AWGN channel. It is represented clearly that the Encryption-OFDM system using proposed Encryption systems 3D-DWT and 3D-DMWTCS gives much better results than OFDM transceiver and the Encryption of 2D-DMWTCS and 2D-DWT OFDM. 


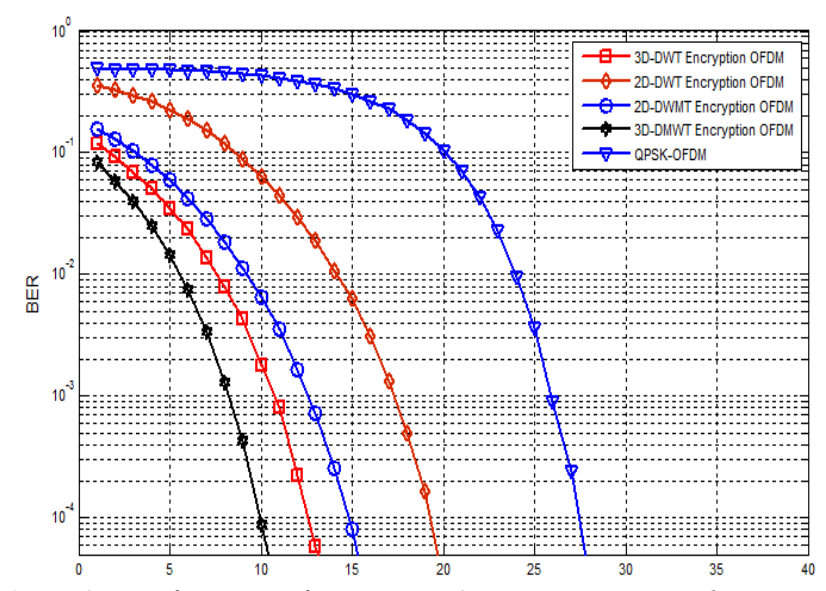

Figure 24. Performance of Encryption-OFDM using proposed Encryption systems in AWGN channel model.

\subsection{The Encryption-OFDM in Flat Fading Channel}

MATLAB V8.1 simulated the results as in Fig. (17) is used here to mimic the results in flat fading channel additional to AWGN excluding a flat fading channel is added to the channel model. For AWGN and flat fading types of channel, the signal is influenced by the fading effect add to AWGN. However, all the frequency assembled of the signal will be influenced with an attenuation and linear distortion for assumed channel and this leads to a Rayleigh's distribution.

The assumption of $10 \mathrm{~Hz}$ is used for Doppler frequency which leads to BER of $10^{-4}$ and the SNR required for encrypted-OFDM using 3D-DWT is about $17 \mathrm{~dB}$ could be seen from Fig (25), while 2D-DWT OFDM scrambling of transceiver is about $23 \mathrm{~dB}$ and the SNR in OFDM transceiver is about $36 \mathrm{~dB}$.

The same thing are shown in from fig.(25) and fig.(26), therefore from fig.(25), fig.(26) and fig.(27) a gain of $19 \mathrm{~dB}$ and $6 \mathrm{~dB}$ for the Encryption-OFDM using 3D-DWT and 3D-DMWTCS Encryption against OFDM 2D-DWT Encryption transceivers are obtained respectively.

Therefore the Encryption-OFDM using 3D-DWT and 3D-DMWTCS outcome bitter results than other Encryption-OFDM system under this model channel.

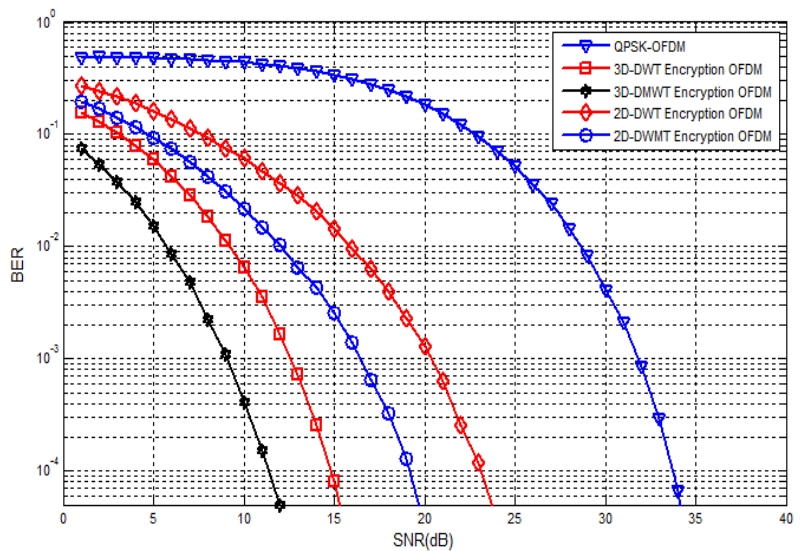

Figure 25. Performance of proposed Encryption systems for Flat Fading Channel with Doppler Shift $=10 \mathrm{~Hz}$.

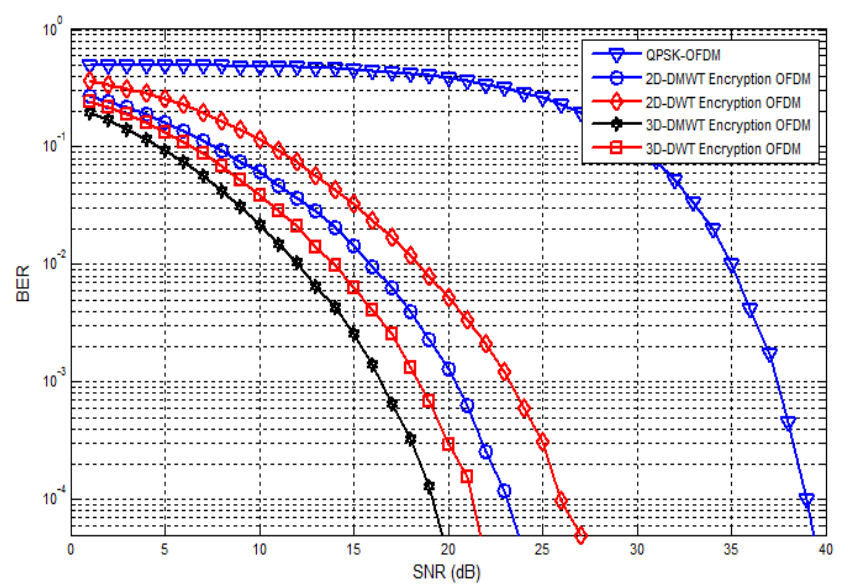

Figure 26. Performance of proposed Encryption systems for Flat Fading Channel with Doppler Shift $=100 \mathrm{~Hz}$.

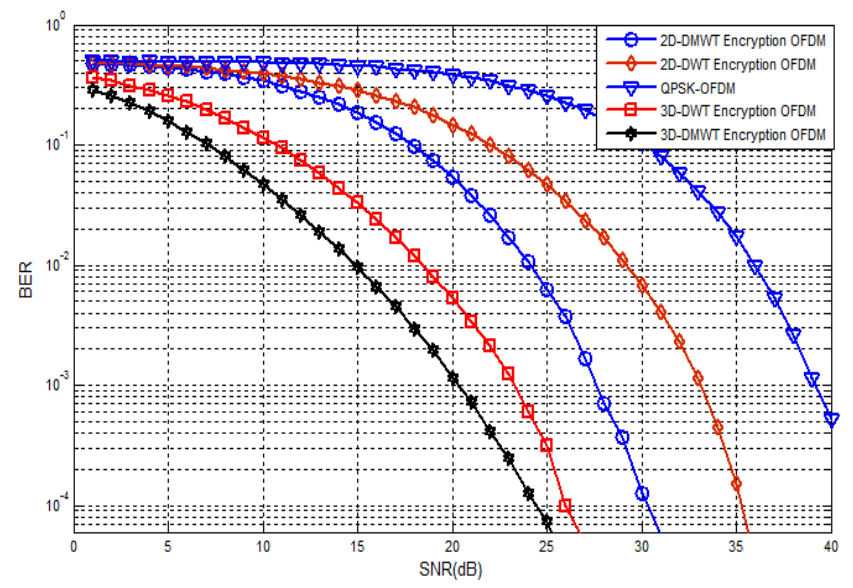

Figure 27. Performance of proposed Encryption systems for Flat Fading Channel with Doppler Shift $=500 \mathrm{~Hz}$.

\subsection{The Encryption-OFDM in Selective Fading Channel}

According to BER performances of Encryption-OFDM using 3D-DMWTCS are mimicked for AWGN with multi-path frequency selective Rayleigh distributed channels. Assuming two ray channel with gain of $-8 \mathrm{~dB}$ for the second path, the second path would have maximum delay of $\tau_{\max }=0.1 \mu \mathrm{sec}$ for range of values of signal to the noise ratio. Fig. (28) represents results at $f_{D \max }=10 \mathrm{~Hz}$ as maximum Doppler shift. It could be seen clearly from Fig.(28) the $\mathrm{BER}=10^{-4}$ would require SNR for Encryption-OFDM using 3D-DWT and 3D-DMWTCS about 19dB, however Encryption-OFDM utilizing 2D-DWT and OFDM transceivers, the SNR are about $31 \mathrm{~dB}$ and $37 \mathrm{~dB}$ respectively. It is clear that a gain of $18 \mathrm{~dB}$ can be obtained by using Encryption-OFDM with 3D-DMWTCS against OFDM transceiver which obtained and about $14 \mathrm{~dB}$ using 3D-DWT. In Figs (28-30) the same thing can noted that Encryption-OFDM using 3D-DMWTCS Encryption system outperforms significantly for this channel model. In this sections the results are briefed in table (4), also those results are computed later by testing the system via transferring approximately $1 \mathrm{M}$ symbols. Table (4) presents SNR values corresponding to BER. 


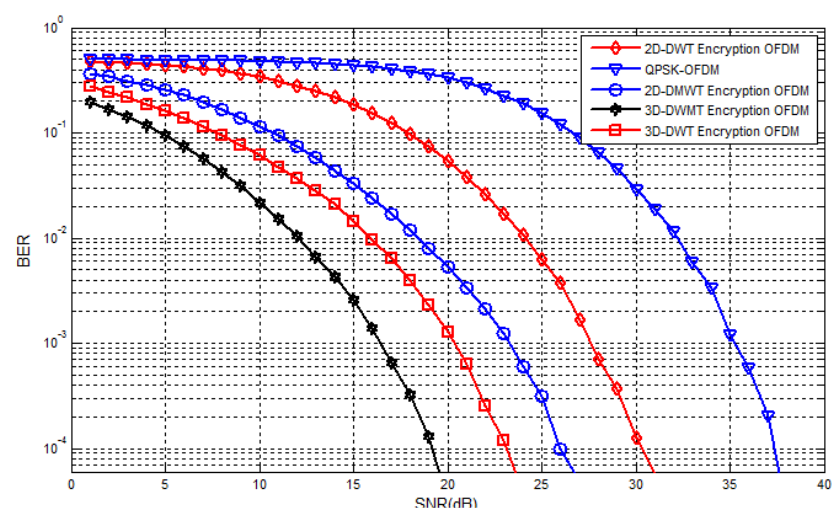

Figure 28. Performance of proposed Encryption systems- for Selective Fading Channel with Max. Doppler Shift $=10 \mathrm{~Hz}$.

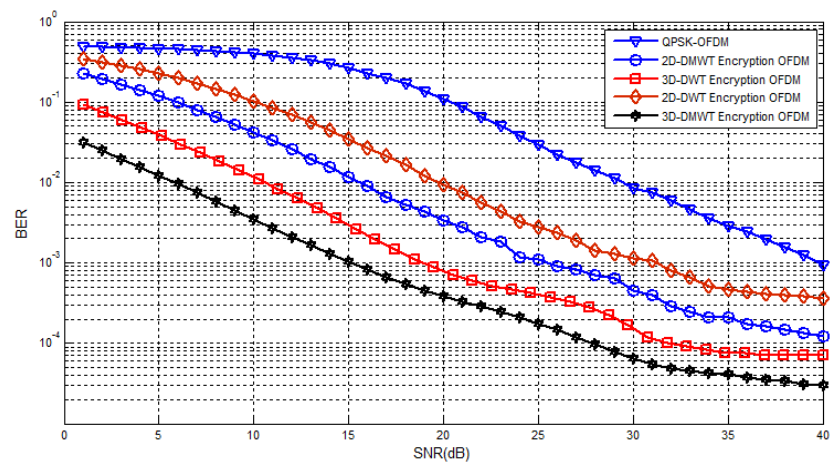

Figure 29. Performance of proposed Encryption systems for Selective Fading Channel with Doppler Shift $=100 \mathrm{~Hz}$.

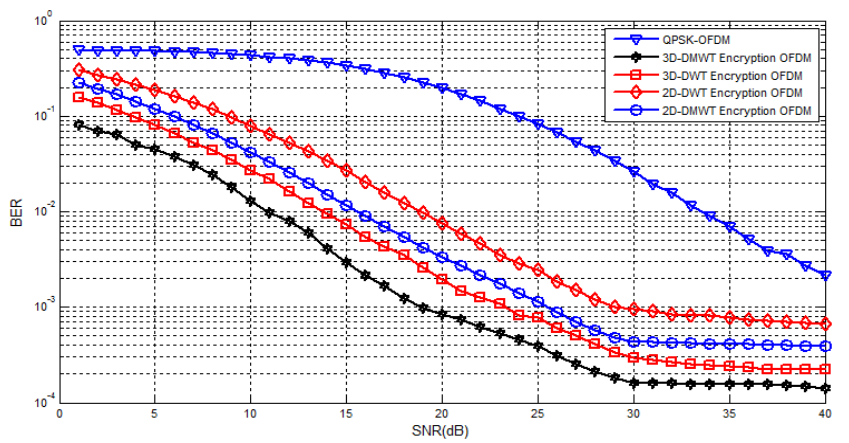

Fig. 30. Performance of proposed Encryption systems for Selective Fading Channel with Doppler Shift $=500 \mathrm{~Hz}$.
Since the essential goal of communication security is the hiding of the fact that a secret message is transmitted, and then it is very important to make the recovered process at receiver. The following interpretations points of this work were present.

1. This work is novel in wireless security based on modern DSP techniques, where most algorithms that were used based on inserting dummy packets or based permutation of Fourier Transform, while in this work, Three Dimensional Transformation based Discrete Multiwavelet Transformation Critically Sampled is adapted and has decrease BER over OFDM modulation in different modulated channels as descripted above.

2. It obvious that the proposed system that based on 3D-DWT and 3D-DMWTCS with permutations is secure against brute force attack, when the required process to retrieve data is governed by permutation preprocess it gives system robust against those kinds of attacks.

3. The key length based on the length of the message which is close to the best security algorithm (One Time Pad) according to what Shannon showed. The length of the key $=16 \times 16 \times 16=4096=2^{12}$.

4. The BER shows great interesting results, the system seems robust and has the immunity for wide range of SNR and this gives two advantages: security and noise immunity.

5. The proposed system fulfills most of the Kerchoff's principles which state that the security of the system based on the assumption that the enemy has full knowledge of the design and implementation details of the security system, i.e. the algorithm could be published in public, only the length of key, permutation algorithm and cipher mode type are kept secret, the resultant message in format will be suitable for transmission, so the system is practically unbreakable, system implementation is easy and it requires a short time.

Table 4. The results for all systems.

\begin{tabular}{|c|c|c|c|c|c|c|c|}
\hline \multirow{3}{*}{ System name } & \multirow{3}{*}{ AWGN } & \multicolumn{3}{|c|}{ Flat Fading } & \multicolumn{3}{|c|}{ Selective Fading } \\
\hline & & \multicolumn{3}{|c|}{ Max. Doppler Shift } & \multicolumn{3}{|c|}{ Max. Doppler Shift } \\
\hline & & $10 \mathrm{~Hz}$ & $100 \mathrm{~Hz}$ & $500 \mathrm{~Hz}$ & $10 \mathrm{~Hz}$ & $100 \mathrm{~Hz}$ & $500 \mathrm{~Hz}$ \\
\hline OFDM- transceiver & 32 & 33 & 39 & non & 37 & non & non \\
\hline 2D-DWT ENCRYPTION- transceiver & 19 & 24 & 32 & 36 & 31 & non & non \\
\hline 2D-DMWTCS ENCRYPTION- transceiver & 15 & 18 & 23 & 31 & 27 & 40 & non \\
\hline 3D-DWT ENCRYPTION- transceiver & 13 & 15 & 21 & 27 & 23 & 33 & non \\
\hline 3D-DMWTCS ENCRYPTION- transceiver & 10 & 12 & 19 & 24 & 18 & 28 & non \\
\hline
\end{tabular}

\section{Conclusion}

The design of Encryption systems are based on SIM908 GSM module. The Encryption processes are implementing based on 2D-DWT, 2D-DMWTCS, 3D-DWT and 3D-DMWTCS transformations. These methods shows that Bandwidth features are preserved and there is no noise expansion, and quality of the recovered was preserved. Also 
the fast algorithms with matrices algebraic for 3D-DWT and 3D-DMWTCS calculation will speeds up the transmitter and receiving data rate by of each square matrices parallel computing; the Inverse transforms are found easily for these transformations, and has the same fast algorithm. The encrypted data is meaningless and thus the residual intelligibility is considerably very low. Permutations are better than inserted dummy components. Cryptanalytic efforts are considerably increased due to altered data components in such away will take infinite time to retrieve data if there is no information how the structure of the encrypted system has been built. These encryption methods also have another benefit that is an increase the immunity of decrypting data sent to the influence of the environment in the channel so improves the BER performance to overall encrypted transceiver as show in the simulation results.

\section{References}

[1] Sim Tech, SIM 908 Hardware Design. V. 2.00. 2012.

[2] ElBahlul, E., Ararab A. "Technical Requirements Of New Framework For GPRS Security Protocol Mobile Banking Application," Procedia Computer Science vol 37 pp 451 - 456, 2014.

[3] S. Irtaza, A .Habib and Q. Islam, "Performance Comparison of LTE Transmission Modes in High Speed Channels Using Soft Sphere Decoder", International Journal of Engineering \& Technology IJET-IJENS Vol.12, pp.73-77, June 2012.

[4] Nakka V.\& Kabirdas A., "Design and realization of augmented reality based navigation assistance system", Intr. J. of computer science and information technologies, Vol.2(6), , pp2842-2846, 2011.

[5] Pani D., Barabino G., Dessì A., Mathieu A., and Raffo L., “A portable realtime monitoring system for kinesitherapic hand rehabilitation exercises," in Proc. 5th Int. Joint Conf. Biomed. Eng. Syst. Technol., pp. 8289, 2012.

[6] D. Evans, "The internet of things, how the next evolution of the internetis changing everything," Cisco Internet Business Solutions Group, Tech.Rep., 2011.

[7] Michel Misiti, Yves Misiti, Georges Oppenheim, Jean-Michel Poggi "Wavelet Toolbox For Use with MATLAB ${ }^{\circledR}$," The MathWorks, Inc, 2002.

[8] Hartono P., Lanny A., Yosephat S. and Rofieko T., " GPS Based Vehicle Tracking Over GPRS For Fleet management And Passenger/ Payload/ Vehicle Security,", ARPN Journal of Engineering and Applied Sciences, vol. 9, no. 11, November 2014.

[9] Ahmed J., "Transform-Domain and DSP Based Secure Speech Communication". Ph.D. dissertation, Hamdard Institute of Information Technology, 2007.

[10] Haruna Matsushita, and Yoshifumi Nishio, "Self-Organizing Map with Weighted Connections Avoiding False-Neighbor Effects ",IEEE international symposium on circuit and systems ISCAS.. pp 2554-2557, June 2010

[11] AM.Kozae, AA Abo Khadra, and T Medhat "Prediction of
Decision for New Data “, IJEST International Journal of Engineering Scienceand Technology. 2010.

[12] Y. Han; X. Que; W. Wang; K. Shuang; M. Huang, "Study on wireless resource consumption of mobile applications in GPRS," 3rd International Conference on Consumer Electronics, Communications and Networks (CECNet), , vol., no., pp.636,640, 20-22 Nov. 2013

[13] Pauls, F.; Krone, S.; Nitzold, W.; Fettweis, G.; Flores, C., "Evaluation of Efficient Modes of Operation of GSM/GPRS Modules for M2M Communications," Vehicular Technology Conference (VTC Fall), 2013 IEEE 78th, vol., no., pp.1,6, 2-5 Sept. 2013

[14] J.-H. Yeh, J.-C. Chen, and C.-C. Lee, "Comparative analysis of energy saving techniques in 3gpp and 3gpp2 systems," Vehicular Technology, IEEE Transactions on, vol. 58, no. 1, pp. 432-448, 2009.

[15] Richard E. Blahut, "Fast Algorithms for Signal Processing," Cambridge University Press, 2010.

[16] John J. Benedetto, "Applied and Numerical Harmonic Analysis: Frames and Bases," Birkha"user Boston, 2008.

[17] S. Mallat. A Theory for Multiresolution Signal Decomposition: the Wavelet Representation. IEEE Transaction on Pattern Analysis and Machine Intelligence, 11, pp. 674-693, 1989 H. Simpson, Dumb Robots, 3rd ed., Springfield: UOS Press, 2004, pp.6-9.

[18] Bruce K., "Multiwavelets for Quantitative Pattern Matching" Proceedings of the 42nd Hawaii International Conference on System Sciences, pp 1- 10, 2009.

[19] M. Weeks, et al, "Discrete Wavelet Transform: Architectures, Design and Performance Issues," Journal of VLSI Signal Processing 35, 155-178, 2003.

[20] Matheel E., Rabab F., "Novel Video Denoising Using 3-D Transformation Techniques," International Journal of Engineering and Advanced Technology (IJEAT), Volume-2, Issue-5, June 2013

[21] Abdul-Rahaim L., Muneer A. " Design of Secure Ad Hoc Network Using Three Dimensional Discrete Wavelet Transformation Based on Performance Enhancement. American Journal of Networks and Communications. Special Issue: Ad Hoc Networks.Vol. 4, No. 3-1, pp. 35-44, 2015.

[22] Galiana-Merino J.J,. Rosa-Herranz J.L, Rosa-Cintas S., and Martinez-Espla J.J.," SeismicWaveTool: Continuous and discrete wavelet analysis and filtering for multichannel seismic data", Computer Physics Communications, Vol. 184, PP. 162-171, 2013 .

[23] N. Sriraam and R. Shyamsunder. "3-D Medical Image Compression Using 3-DWavelet Coders", Digital Signal Processing, 21(1):100-109, January 2011.

[24] Al-Taai H. N., "Computationally efficient wavelet based algorithms for optical flow estimation ", Ph.D. Thesis, Univ. of Technology, Electrical and electronic engineering, Dep., Oct.2005.

[25] El-Anzy L., " Design and Simulation of STBC-(OFDM and CDMA) Transceivers based on Hybrid Transforms ", Ph.D. Thesis, Univ. of Technology, Electrical and electronic engineering, Dep., Oct.2006. 